\title{
On the Secrecy Rate of Downlink NOMA in Underlay Spectrum Sharing with Imperfect CSI
}

\author{
Invited Paper
}

\author{
Vaibhav Kumar*, Mark F. Flanagan*, Daniel Benevides da Costa ${ }^{\dagger}$, and Le-Nam Tran* \\ *School of Electrical and Electronic Engineering, University College Dublin, Belfield, Dublin 4, Ireland \\ ${ }^{\dagger}$ Future Technology Research Center, National Yunlin University of Science and Technology, Douliu, Yunlin 64002, Taiwan \\ Email: \{vaibhav.kumar, mark.flanagan, danielbcosta\}@ieee.org, nam.tran@ucd.ie
}

\begin{abstract}
In this paper, we present the ergodic sum secrecy rate (ESSR) analysis of an underlay spectrum sharing nonorthogonal multiple access (NOMA) system. We consider the scenario where the power transmitted by the secondary transmitter (ST) is constrained by the peak tolerable interference at multiple primary receivers (PRs) as well as the maximum transmit power of the ST. The effect of channel estimation error is also taken into account in our analysis. We derive exact and asymptotic closedform expressions for the ESSR of the downlink NOMA system, and show that the performance can be classified into two distinct regimes, i.e., it is dictated either by the interference constraint or by the power constraint. Our results confirm the superiority of the NOMA-based system over its orthogonal multiple access (OMA) based counterpart. More interestingly, our results show that NOMA helps in maintaining the secrecy rate of the strong user while significantly enhancing the secrecy performance of the weak user as compared to OMA. The correctness of the proposed investigation is corroborated through Monte Carlo simulation.
\end{abstract}

\section{INTRODUCTION}

Non-orthogonal multiple access (NOMA) has gained tremendous attention as a potential multiple access technology for the next-generation wireless systems. It is proven to be capable of providing massive connectivity, low latency and higher achievable rate as compared to the traditional orthogonal multiple access (OMA) system [1]. In order to serve multiple users using a given resource block (time slot, frequency band, and/or spreading code), NOMA uses powerdivision multiplexing at the transmitter's side and successive interference cancellation (SIC) at the receivers' side. Underlay spectrum sharing is another potential technology to mitigate the problem of spectrum scarcity, where an unlicensed/secondary network simultaneously uses the spectrum owned by a licensed/primary network in such a manner that the interference inflicted by the secondary network on the primary one remains below a certain threshold [2]. The benefits of underlay spectrum sharing NOMA systems over their OMAbased counterparts were discussed in [3]-[5].

In today's privacy-concerned society, the broadcast nature of radio waves poses a significant risk that wireless communications may be intercepted by an eavesdropper. Therefore, physical-layer security (PLS), which provides an additional layer of security from an information-theoretic perspective, has

\footnotetext{
This publication has emanated from research conducted with the financial support of Science Foundation Ireland (SFI) and is co-funded under the European Regional Development Fund under Grant Number 17/CDA/4786.
}

gained significant attention in the past couple of decades [6]. The secrecy outage probability (SOP) analysis of a multiplerelay-assisted two-user downlink NOMA system was presented in [7], where the authors proposed different relay selection schemes to enhance the secrecy performance of the system. The ergodic sum secrecy rate (ESSR) analysis of a two-user downlink and uplink cooperative NOMA system with untrusted relaying was performed in [8], while the authors in [9] presented the ergodic secrecy rate (ESR) and SOP analysis of a full-duplex relay-assisted two-user downlink NOMA system with energy harvesting.

On the other hand, some recent contributions on the secrecy analysis of underlay spectrum sharing systems include [10][12]. Specifically, the secrecy throughput and energy efficiency analysis of a spectrum sharing system consisting of one primary source-destination pair, multiple secondary sourcedestination pairs and an eavesdropper was presented in [10], where primary and secondary networks either interfere or cooperate with each other in order to improve the secrecy performance. In [11], the authors derived closed-form expressions for the non-zero secrecy rate, ESR and SOP of an outage-constrained spectrum sharing system with transmitter selection and unreliable backhaul. The analysis of the achievable secrecy rate for an underlay spectrum sharing multiuser massive multiple-input multiple-output (mMIMO) system was presented in [12]. However, to the best of the authors' knowledge, the secrecy rate analysis of an underlay spectrum sharing system where the secondary transmitter communicates with the secondary receivers using NOMA has not yet been investigated in the literature. Therefore, in this paper, we present the ESSR analysis of a two-user downlink NOMA system in an underlay spectrum sharing scenario. The main contributions in this paper are listed below:

- We derive an exact closed-form expression for the ESSR of a downlink NOMA system in an underlay spectrum sharing scenario with multiple primary receivers (PRs) considering the effect of imperfect channel state information (CSI). We show the effect of the peak tolerable interference power at the PRs, the maximum power budget at the secondary transmitter (ST), channel estimation error and the number of PRs on the ESSR of the NOMA system. 


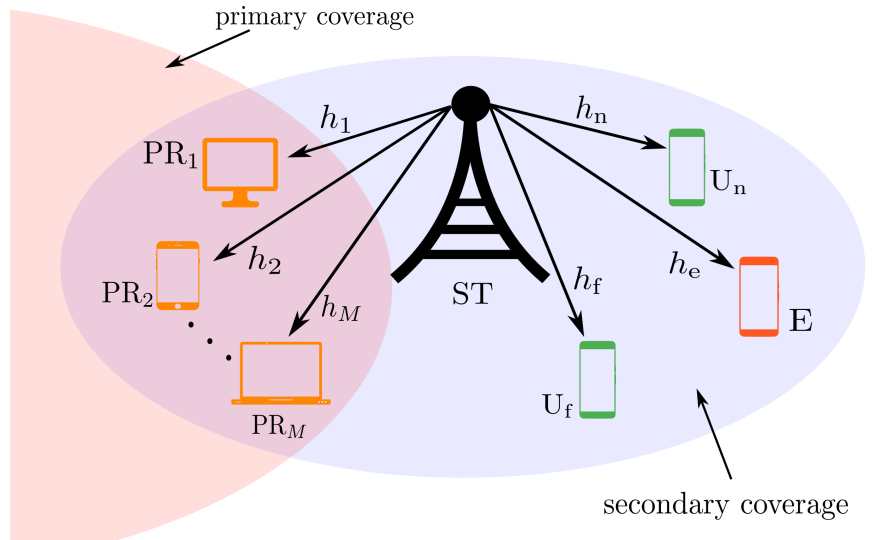

Fig. 1. System model for underlay spectrum sharing NOMA with multiple primary receivers.

- We derive an asymptotic expression for the ESSR of the downlink NOMA system for large values of peak tolerable interference at the PRs. The asymptotic analysis confirms that the slope of the ESSR tends to zero (w.r.t. the peak tolerable interference at the PRs), and the ESSR becomes independent of the number of PRs as well as the quality of the channel between the ST and PRs.

- Using the exact closed-form expressions for the ESR, we perform a one-dimensional numerical search to allocate power between the NOMA users in such a manner that the ESR of the strong user in the NOMA system and the corresponding OMA system becomes equal, and then the remaining power is allocated to the weak NOMA user. Such a power allocation policy ensures that the secrecy performance of the strong user in the NOMA system is the same as that of the strong user in the corresponding OMA system while the secrecy performance of the weak user in the NOMA system is enhanced significantly as compared to that in the OMA system, resulting in a performance superiority of the NOMA system over its OMA-based counterpart in terms of ESSR.

\section{SYSTEM MODEL}

Consider an underlay spectrum sharing system as shown in Fig. 1, consisting of $M$ PRs $\left(\mathrm{PR}_{1}, \mathrm{PR}_{2}, \ldots, \mathrm{PR}_{M}\right)$, one $\mathrm{ST}$, two ${ }^{1}$ secondary (NOMA) receivers, denoted by $\mathrm{U}_{\mathrm{n}}$ (near user) and $\mathrm{U}_{\mathrm{f}}$ (far user), and one eavesdropper E. It is assumed that all nodes are single-antenna devices. The distances of $\mathrm{PR}_{m}(m \in\{1,2, \ldots, M\}), \mathrm{U}_{\mathrm{n}}, \mathrm{U}_{\mathrm{f}}$ and $\mathrm{E}$ from the $\mathrm{ST}$ are denoted by $d_{m}, d_{\mathrm{n}}, d_{\mathrm{f}}$ and $d_{\mathrm{e}}$, respectively. The channel fading coefficient for ST-PR ${ }_{m}$, ST-U $\mathrm{U}_{\mathrm{n}}, \mathrm{ST}-\mathrm{U}_{\mathrm{f}}$ and ST-E links are, respectively, given by $h_{m} \sim \mathcal{C N}\left(0, \Omega_{m}=d_{m}^{-\alpha}\right)$, $h_{\mathrm{n}} \sim \mathcal{C N}\left(0, \Omega_{\mathrm{n}}=d_{\mathrm{n}}^{-\alpha}\right), h_{\mathrm{f}} \sim \mathcal{C N}\left(0, \Omega_{\mathrm{f}}=d_{\mathrm{f}}^{-\alpha}\right)$ and $h_{\mathrm{e}} \sim \mathcal{C N}\left(0, \Omega_{\mathrm{e}}=d_{\mathrm{e}}^{-\alpha}\right)$, with $\alpha$ denoting the path loss

\footnotetext{
${ }^{1}$ As suggested by the 3GPP-LTE, one resource block should be allocated for two NOMA users. Therefore, in this paper, we consider only two downlink NOMA users. Similar assumptions were considered in [7]-[9]. However, our analysis can be extended to multiple downlink NOMA users by adopting the user pairing scheme proposed in [9].
}

exponent. The corresponding channel gain is given by $g_{i} \triangleq$ $\left|h_{i}\right|^{2}, i \in\{1,2, \ldots, M, \mathrm{n}, \mathrm{f}, \mathrm{e}\}$. We assume that the ST has imperfect instantaneous CSI of the ST-PR ${ }_{m}$, ST-Un, ST$\mathrm{U}_{\mathrm{f}}$ and ST-E links. Let the estimate of $h_{i}$ be denoted by $\tilde{h}_{i}$, and $h_{i}=\tilde{h}_{i}+\epsilon_{i}$, where $\epsilon_{i} \sim \mathcal{C N}\left(0, \sigma_{\epsilon}^{2}\right)$ is the channel estimation error. Therefore, $\tilde{h}_{i} \sim \mathcal{C N}\left(0, \tilde{\Omega}_{i}\right)$, where $\tilde{\Omega}_{i}=\Omega_{i}-\sigma_{\epsilon}^{2}$ and the corresponding channel gain is denoted by $\tilde{g}_{i}$. The peak (instantaneous) interference that the PRs can tolerate from the secondary network is denoted by $I_{\mathrm{p}}$, and the maximum transmit power at the $\mathrm{ST}$ is denoted by $P_{\max }$. Therefore, the instantaneous transmit power of the ST is given by $P=\min \left\{P_{\max }, \frac{I_{\mathrm{p}}}{\tilde{g}_{1}}, \frac{I_{\mathrm{p}}}{\tilde{g}_{2}}, \ldots, \frac{I_{\mathrm{p}}}{\tilde{g}_{M}}\right\}=\min \left\{P_{\max }, \frac{I_{\mathrm{p}}}{\tilde{g}_{\mathrm{p}}}\right\}$, where $\tilde{g}_{\mathrm{p}} \triangleq \max _{m} \tilde{g}_{m}$. We denote the probability density function (PDF), cumulative distribution function (CDF) and complementary $\mathrm{CDF}$ (CCDF) of a random variable $\mathcal{X}$ by $f_{\mathcal{X}}(\cdot), F_{\mathcal{X}}(\cdot)$ and $\mathcal{F}_{\mathcal{X}}(\cdot)$, respectively, and we define $\mathscr{F}_{\mathcal{X}}\left(c_{1}, c_{2}\right) \triangleq \int_{c_{1}}^{c_{2}} f_{\mathcal{X}}(t) \mathrm{d} t$.

\section{ANALYSIS OF THE ESSR}

Based on the estimated CSI at the ST, the users $U_{n}$ and $U_{f}$ are further categorized as $\mathrm{U}_{\mathrm{s}}$ (strong user) and $\mathrm{U}_{\mathrm{w}}$ (weak user), where $\mathrm{s} \triangleq \operatorname{argmax}_{j \in\{\mathrm{n}, \mathrm{f}\}} \tilde{g}_{j}$ and $\mathrm{w} \triangleq \operatorname{argmin}_{j \in\{\mathrm{n}, \mathrm{f}\}} \tilde{g}_{j}$. The signal received at $\mathrm{U}_{k}, k \in\{\mathrm{s}, \mathrm{w}\}$ is given by

$$
y_{k}=\left(\tilde{h}_{k}+\epsilon_{k}\right)\left(\sqrt{a_{\mathrm{s}} P} x_{\mathrm{s}}+\sqrt{a_{\mathrm{w}} P} x_{\mathrm{w}}\right)+n_{k},
$$

where $x_{k}$ is the unit-energy information-bearing complex constellation symbol and $n_{k} \sim \mathcal{C N}(0,1)$ is the additive white Gaussian noise (AWGN) at node $\mathrm{U}_{k}$. Following the NOMA principle, it is assumed that $a_{\mathrm{s}}<a_{\mathrm{w}}$ and $a_{\mathrm{s}}+a_{\mathrm{w}}=1$. The weak user decodes $x_{\mathrm{w}}$ by treating the interference due to $x_{\mathrm{s}}$ as additional noise, whereas the strong user first decodes $x_{\mathrm{w}}$ by treating the interference due to $x_{\mathrm{s}}$ as additional noise and then applies successive interference cancellation (SIC) to decode $x_{\mathrm{s}}$. Therefore, the signal-to-interference-plus-noise ratio (SINR) at $\mathrm{U}_{\mathrm{s}}$ and $\mathrm{U}_{\mathrm{w}}$ are, respectively, given by

$$
\gamma_{\mathrm{s}}=\frac{a_{\mathrm{s}} \tilde{g}_{\mathrm{s}} P}{1+\sigma_{\epsilon}^{2} P}, \quad \gamma_{\mathrm{w}}=\frac{a_{\mathrm{w}} \tilde{g}_{\mathrm{w}} P}{1+a_{\mathrm{s}} \tilde{g}_{\mathrm{w}} P+\sigma_{\epsilon}^{2} P} .
$$

Following [13, Ch. 15], we consider the case that E follows the same decoding order as that of the legitimate users. Therefore, the SINR at E for decoding $x_{\mathrm{w}}$ and $x_{\mathrm{s}}$ are respectively given by

$$
\gamma_{\mathrm{e}, \mathrm{w}}=\frac{a_{\mathrm{w}} \tilde{g}_{\mathrm{e}} P}{1+a_{\mathrm{s}} \tilde{g}_{\mathrm{e}} P+\sigma_{\epsilon}^{2} P}, \quad \gamma_{\mathrm{e}, \mathrm{s}}=\frac{a_{\mathrm{s}} \tilde{g}_{\mathrm{e}} P}{1+\sigma_{\epsilon}^{2} P} .
$$

Let $\hat{g}_{\mathrm{s}} \triangleq \sigma_{\epsilon}^{2}+a_{\mathrm{s}} \tilde{g}_{\mathrm{s}}, \hat{g}_{\mathrm{w}} \triangleq \sigma_{\epsilon}^{2}+a_{\mathrm{s}} \tilde{g}_{\mathrm{w}}, \check{g}_{\mathrm{w}} \triangleq \sigma_{\epsilon}^{2}+\tilde{g}_{\mathrm{w}}$ and $\hat{g}_{\mathrm{e}} \triangleq \sigma_{\epsilon}^{2}+a_{\mathrm{s}} \tilde{g}_{\mathrm{e}}$. Then using the standard statistical procedure of transformation of random variables, it follows that

$$
\begin{aligned}
& f_{\hat{g}_{\mathrm{s}}}(x)=\sum_{\ell \in\{1,2,3\}} \hat{A}_{\ell} \exp \left(\frac{-x}{a_{\mathrm{s}} \Xi_{\ell}}\right), \mathcal{F}_{\hat{g}_{\mathrm{s}}}(x)=\sum_{\ell \in\{1,2,3\}} \hat{\mathcal{A}}_{\ell} \exp \left(\frac{-x}{a_{\mathrm{s}} \Xi_{\ell}}\right), \\
& f_{\hat{g}_{\mathrm{w}}}(x)=-\hat{A}_{2} \exp \left(\frac{-x}{a_{\mathrm{s}} \Xi_{2}}\right), \mathcal{F}_{\hat{g}_{\mathrm{w}}}(x)=-\hat{\mathcal{A}}_{2} \exp \left(\frac{-x}{a_{\mathrm{s}} \Xi_{2}}\right),
\end{aligned}
$$




$$
\begin{gathered}
f_{\check{g}_{\mathrm{w}}}(x)=\check{A}_{2} \exp \left(\frac{-x}{\Xi_{2}}\right), \mathcal{F}_{\check{g}_{\mathrm{w}}}(x)=\check{\mathcal{A}}_{2} \exp \left(\frac{-x}{\Xi_{2}}\right), \\
f_{\hat{g}_{\mathrm{e}}}(x)=\hat{C}_{\mathrm{e}} \exp \left(\frac{-x}{a_{\mathrm{s}} \tilde{\Omega}_{\mathrm{e}}}\right), \mathscr{F}_{\hat{g}_{\mathrm{e}}}\left(\sigma_{\epsilon}^{2}, x\right)=1-\hat{\mathscr{C}}_{\mathrm{e}} \exp \left(\frac{-x}{a_{\mathrm{s}} \tilde{\Omega}_{\mathrm{e}}}\right), \\
\mathscr{F}_{\check{g}_{\mathrm{e}}}\left(\sigma_{\epsilon}^{2}, x\right)=1-\check{\mathscr{C}}_{\mathrm{e}} \exp \left(\frac{-x}{\tilde{\Omega}_{\mathrm{e}}}\right),
\end{gathered}
$$

where $\Xi_{1}=\tilde{\Omega}_{\mathrm{n}}, \quad \Xi_{2}=\tilde{\Omega}_{\mathrm{n}, \mathrm{f}}, \quad \Xi_{3}=\tilde{\Omega}_{\mathrm{f}}, \quad \tilde{\Omega}_{\mathrm{n}, \mathrm{f}}=$ $\left(\tilde{\Omega}_{\mathrm{n}}^{-1}+\tilde{\Omega}_{\mathrm{f}}^{-1}\right)^{-1}, \hat{A}_{\ell}=\frac{(-1)^{\ell+1}}{a_{\mathrm{s}} \Xi_{\ell}} \exp \left(\frac{\sigma_{\epsilon}^{2}}{a_{\mathrm{s}} \Xi_{\ell}}\right), \hat{\mathcal{A}}_{\ell}=a_{\mathrm{s}} \Xi_{\ell} \hat{A}_{\ell}$, $\check{A}_{2}=\frac{1}{\Xi_{2}} \exp \left(\frac{\sigma_{\epsilon}^{2}}{\Xi_{2}}\right), \check{\mathcal{A}}_{2}=\Xi_{2} \check{A}_{2}, \hat{C}_{\mathrm{e}}=\frac{1}{a_{\mathrm{s}} \tilde{\Omega}_{\mathrm{e}}} \exp \left(\frac{\sigma_{\epsilon}^{2}}{a_{\mathrm{s}} \tilde{\Omega}_{\mathrm{e}}}\right)$ and $\hat{\mathscr{C}}_{\mathrm{e}}=a_{\mathrm{s}} \tilde{\Omega}_{\mathrm{e}} \hat{C}_{\mathrm{e}}$. From [14], the PDF of $\tilde{g}_{\mathrm{p}}$ is given by

$$
f_{\tilde{g}_{\mathrm{p}}}(x)=-\sum_{\boldsymbol{\eta} \in \Phi} \kappa_{\boldsymbol{\eta}} B_{\boldsymbol{\eta}} \exp \left(-B_{\boldsymbol{\eta}} x\right),
$$

where $\Phi=\left\{\boldsymbol{\eta}=\left[\eta_{1} \eta_{2} \cdots \eta_{M}\right] \in \mathbb{Z}_{2}^{M} \mid \sum_{m=1}^{M} \eta_{m}>0\right\}$ represents the set of all nonzero binary vectors of length $M, \mathbb{Z}_{2}=$ $\{0,1\}, \kappa_{\boldsymbol{\eta}}=\prod_{m=1}^{M}(-1)^{\eta_{m}}$ and $B_{\boldsymbol{\eta}}=\sum_{m=1}^{M}\left(\eta_{m} / \tilde{\Omega}_{m}\right)$.

Finally, the ESR of the strong user can be written as

$$
\begin{aligned}
\bar{R}_{\mathrm{s}} & =\mathbb{E}\left\{\max \left\{\log _{2}\left(1+\gamma_{\mathrm{s}}\right)-\log _{2}\left(1+\gamma_{\mathrm{e}, \mathrm{s}}\right), 0\right\}\right\} \\
& =\mathbb{E}\left\{\max \left\{\log _{2}\left(1+\hat{g}_{\mathrm{s}} P\right)-\log _{2}\left(1+\hat{g}_{\mathrm{e}} P\right), 0\right\}\right\} .
\end{aligned}
$$

Theorem 1. A closed-form expression for the ESR of $\mathrm{U}_{\mathrm{s}}$ can be derived as

$$
\bar{R}_{\mathrm{s}}=\mathscr{I}_{\mathrm{s}, 1}+\mathscr{I}_{\mathrm{s}, 2}-\mathscr{I}_{\mathrm{s}, 3}-\mathscr{I}_{\mathrm{s}, 4}
$$

where the expressions for $\mathscr{I}_{\mathrm{s}, 1}, \mathscr{I}_{\mathrm{s}, 2}, \mathscr{I}_{\mathrm{s}, 3}$ and $\mathscr{I}_{\mathrm{s}, 4}$ are given by $(3)-(6)$, shown at the top of the next page, $\Theta \triangleq I_{\mathrm{p}} / P_{\max }$, $\Xi_{\ell, e} \triangleq\left(\Xi_{\ell}^{-1}+\tilde{\Omega}^{-1}\right)^{-1}$, and $\operatorname{Ei}(\cdot)$ and $\mathrm{E}_{1}(\cdot)$ are the exponential integrals.

Proof: See Appendix A.

On the other hand, the ESR for the weak user $U_{w}$ can be written as

$$
\begin{aligned}
\bar{R}_{\mathrm{w}}=\mathbb{E}\left\{\max \left\{\log _{2}\left(1+\gamma_{\mathrm{w}}\right)-\log _{2}\left(1+\gamma_{\mathrm{e}, \mathrm{w}}\right), 0\right\}\right\} \\
=\mathbb{E}\left\{\operatorname { m a x } \left\{\log _{2}\left(\frac{1+\left(\tilde{g}_{\mathrm{w}}+\sigma_{\epsilon}^{2}\right) P}{1+\left(a_{\mathrm{s}} \tilde{g}_{\mathrm{w}}+\sigma_{\epsilon}^{2}\right) P}\right)\right.\right. \\
\left.\left.\quad-\log _{2}\left(\frac{1+\left(\tilde{g}_{\mathrm{e}}+\sigma_{\epsilon}^{2}\right) P}{1+\left(a_{\mathrm{s}} \tilde{g}_{\mathrm{e}}+\sigma_{\epsilon}^{2}\right) P}\right), 0\right\}\right\} .
\end{aligned}
$$

Theorem 2. A closed-form expression for the ESR of $\mathrm{U}_{\mathrm{w}}$ can be derived as

$$
\bar{R}_{\mathrm{w}}=\mathscr{I}_{\mathrm{w}, 1}+\mathscr{I}_{\mathrm{w}, 2}-\mathscr{I}_{\mathrm{w}, 3}-\mathscr{I}_{\mathrm{w}, 4}
$$

where the expressions for $\mathscr{I}_{\mathrm{w}, 1}, \mathscr{I}_{\mathrm{w}, 2}, \mathscr{I}_{\mathrm{w}, 3}$ and $\mathscr{I}_{\mathrm{w}, 4}$ are given by (11) - (14), shown on the next page.

Proof: See Appendix B.

Using (2) and (10), a closed-form expression for the ESSR of the NOMA system is given by $\bar{R}_{\text {sum }}=\bar{R}_{\mathrm{s}}+\bar{R}_{\mathrm{w}}$.
ESSR for OMA: For the case of an underlay spectrum sharing OMA system, the ST transmits $\sqrt{P} x_{\mathrm{s}}$ to $\mathrm{U}_{\mathrm{s}}$ in the first time slot, and $\sqrt{P} x_{\mathrm{w}}$ to $\mathrm{U}_{\mathrm{w}}$ in the second time slot. For a fair comparison between the NOMA and OMA systems, we consider the same value of $I_{\mathrm{p}}$ for both systems. Therefore, the ESSR for the OMA system is given by

$$
\begin{aligned}
& \bar{R}_{\text {sum }, \text { OMA }}=\bar{R}_{\mathrm{s}, \text { OMA }}+\bar{R}_{\mathrm{w}, \text { OMA }} \\
& =\sum_{k \in\{\mathrm{s}, \mathrm{w}\}} \mathbb{E}\left\{0.5 \max \left\{\log _{2}\left(\frac{1+\tilde{g}_{k} P}{1+\tilde{g}_{\mathrm{e}} P}\right), 0\right\}\right\} .
\end{aligned}
$$

As the focus of this paper is on the NOMA-based system, we do not provide the closed-form expression for $\bar{R}_{\text {sum,OMA }}$. Next, we carry out the asymptotic analysis $\left(I_{\mathrm{p}} \rightarrow \infty\right)$ of the ESSR for the NOMA system.

\section{ASYMPTOTIC ESSR FOR THE NOMA SYSTEM}

Theorem 3. An analytical expression for the asymptotic $\left(I_{\mathrm{p}} \rightarrow \infty\right)$ ESSR for the NOMA system can be derived as

$$
\bar{R}_{\text {sum }, \text { asymp }}=\mathcal{I}_{\mathrm{s}}+\mathcal{I}_{\mathrm{w}},
$$

where the closed-form expressions for $\mathcal{I}_{\mathrm{s}}$ and $\mathcal{I}_{\mathrm{w}}$ are given by (17) and (18), respectively, shown on the next page.

Proof: Note that for $I_{\mathrm{p}} \rightarrow \infty$ and finite $P_{\max }$, we have $\Theta=\frac{I_{\mathrm{P}}}{P_{\max }} \rightarrow \infty$. Letting $\Theta \rightarrow \infty$ in (19) and (20), and then solving the integrals following a similar line of argument as given in Appendix $\mathrm{A}$, a closed-form expression for $\bar{R}_{\text {sum,asymp }}$ is given by (16). This concludes the proof.

It can be noted from (16) - (18) that for large values of $I_{\mathrm{p}}$, the ESSR of the NOMA is independent of the number of PRs as well as the quality of the link between the ST and PRs. Also, the slope of the ESSR w.r.t $I_{\mathrm{p}}$ becomes equal to zero for $I_{\mathrm{p}} \rightarrow \infty$.

\section{Results AND Discussions}

In this section, we present the numerical and analytical results for the ESSR of NOMA and OMA systems. We consider a system with $d_{\mathrm{n}}=30 \mathrm{~m}, d_{\mathrm{f}}=100 \mathrm{~m}, d_{\mathrm{e}}=150 \mathrm{~m}$, $\left\{d_{1}, d_{2}, d_{3}, d_{4}\right\}=\{200,205,210,215\} \mathrm{m}, \sigma_{\epsilon}^{2}=2 \times 10^{-5}$, and $\alpha=2$, unless stated otherwise. In the case of NOMA, we use the bisection method to find the value of $a_{\mathrm{s}}$ such that $\bar{R}_{\mathrm{S}}=\bar{R}_{\mathrm{s}, \mathrm{OMA}}$, and then the remaining power fraction $\left(a_{\mathrm{w}}\right)$ is allocated to the weak user. In each figure, the legend indicates the numerically obtained results, while the solid curves indicate the analytical results.

Fig. 2 shows a performance comparison of the ESR of the NOMA and OMA systems. It can be observed from the figure that the ESR of $U_{s}$ in the NOMA system is equal to that in the case of the OMA system (by virtue of the power allocation scheme), whereas the ESR of $\mathrm{U}_{\mathrm{w}}$ in the NOMA system is higher that that of the OMA system. This result confirms that NOMA helps in maintaining the secrecy rate of the strong user while significantly enhancing the secrecy performance of the weak user as compared to OMA. We also show the asymptotic $\left(I_{\mathrm{p}} \rightarrow \infty\right)$ ESR for the NOMA system, which matches well with the exact ESR for large $I_{\mathrm{p}}$. Moreover, a close agreement 


$$
\begin{gathered}
\mathscr{I}_{\mathrm{s}, 1}=-\log _{2}(e) a_{\mathrm{s}}\left[\sum_{\ell \in\{1,2,3\}} \hat{A}_{\ell}\left\{\Xi_{\ell} \mathbb{J}_{1}\left(a_{\mathrm{s}} \Xi_{\ell}\right)-\hat{\mathscr{C}}_{\mathrm{e}} \Xi_{\ell, \mathrm{e}} \mathbb{J}_{1}\left(a_{\mathrm{s}} \Xi_{\ell, \mathrm{e}}\right)\right\}\right]\left[\sum_{\boldsymbol{\eta} \in \Phi} \kappa_{\boldsymbol{\eta}}\left\{1-\exp \left(-B_{\boldsymbol{\eta}} \Theta\right)\right\}\right] \\
\mathscr{I}_{\mathrm{s}, 2}=-\log _{2}(e) a_{\mathrm{s}} \sum_{\ell \in\{1,2,3\}} \sum_{\boldsymbol{\eta} \in \Phi} \hat{A}_{\ell} \kappa_{\eta}\left[\Xi_{\ell} \mathbb{J}_{2}\left(a_{\mathrm{s}} \Xi_{\ell}\right)-\hat{\mathscr{C}}_{\mathrm{e}} \Xi_{\ell, \mathrm{e}} \mathbb{J}_{2}\left(a_{\mathrm{s}} \Xi_{\ell, \mathrm{e}}\right)\right] \\
\mathscr{I}_{\mathrm{s}, 3}=-\log _{2}(e) a_{\mathrm{s}} \hat{C}_{\mathrm{e}}\left[\sum_{\ell \in\{1,2,3\}} \hat{\mathcal{A}}_{\ell} \Xi_{\ell, \mathrm{e}} \mathbb{J}_{1}\left(a_{\mathrm{s}} \Xi_{\ell, \mathrm{e}}\right)\right]\left[\sum_{\boldsymbol{\eta} \in \Phi} \kappa_{\boldsymbol{\eta}}\left\{1-\exp \left(-B_{\boldsymbol{\eta}} \Theta\right)\right\}\right] \\
\mathscr{I}_{\mathrm{s}, 4}=-\log _{2}(e) a_{\mathrm{s}} \hat{C}_{\mathrm{e}} \sum_{\ell \in\{1,2,3\}} \sum_{\boldsymbol{\eta} \in \Phi} \hat{\mathcal{A}}_{\ell} \kappa_{\boldsymbol{\eta}} \Xi_{\ell, 2} \mathbb{J}_{2}\left(a_{\mathrm{s}} \Xi_{\ell, \mathrm{e}}\right) \\
\mathbb{J}_{1}(\Omega)=\exp \left(\frac{-\sigma_{\epsilon}^{2}}{\Omega}\right) \ln \left(1+\sigma_{\epsilon}^{2} P_{\max }\right)+\exp \left(\frac{1}{\Omega P_{\max }}\right) \mathrm{E}_{1}\left[\frac{1}{\Omega}\left(\frac{1}{P_{\mathrm{max}}}+\sigma_{\epsilon}^{2}\right)\right] \\
\mathbb{J}_{2}(\Omega) \triangleq \exp \left(\frac{-\sigma_{\epsilon}^{2}}{\Omega}\right) \operatorname{Ei}\left(-B_{\boldsymbol{\eta}} \Theta\right)-\frac{B_{\boldsymbol{\eta}}^{-1}}{B_{\boldsymbol{\eta}}^{-1}-I_{\mathrm{p}} \Omega}\left\{\exp \left(I_{\mathrm{p}} \sigma_{\epsilon}^{2} B_{\boldsymbol{\eta}}-\frac{\sigma_{\epsilon}^{2}}{\Omega}\right) \operatorname{Ei}\left(-B_{\boldsymbol{\eta}}\left(\Theta+I_{\mathrm{p}} \sigma_{\epsilon}^{2}\right)\right)-\exp \left(\frac{\Theta}{I_{\mathrm{p}} \Omega}-B_{\boldsymbol{\eta}} \Theta\right)\right. \\
\left.\times \operatorname{Ei}\left(\frac{-\Theta-I_{\mathrm{p}} \sigma_{\epsilon}^{2}}{I_{\mathrm{p}} \Omega}\right)\right\}+\exp \left(-B_{\boldsymbol{\eta}} \Theta-\frac{\sigma_{\epsilon}^{2}}{\Omega}\right)\left\{\ln \left(1+\frac{I_{\mathrm{p}} \sigma_{\epsilon}^{2}}{\Theta}\right)+\exp \left(\frac{\Theta+I_{\mathrm{p}} \sigma_{\epsilon}^{2}}{I_{\mathrm{p}} \Omega}\right) \mathrm{E}_{1}\left(\frac{\Theta+I_{\mathrm{p}} \sigma_{\epsilon}^{2}}{I_{\mathrm{p}} \Omega}\right)\right\}
\end{gathered}
$$

$$
\begin{gathered}
\mathscr{I}_{\mathrm{w}, 1}=-\log _{2}(e) \sum_{\boldsymbol{\eta} \in \Phi} \kappa_{\boldsymbol{\eta}}\left\{1-\exp \left(-B_{\boldsymbol{\eta}} \Theta\right)\right\}\left[\check{A}_{2} \Xi_{2} \mathbb{J}_{1}\left(\Xi_{2}\right)-\check{A}_{2} \check{\mathscr{C}}_{\mathrm{e}} \Xi_{2, \mathrm{e}} \mathbb{J}_{1}\left(\Xi_{2, \mathrm{e}}\right)+\hat{A}_{2} a_{\mathrm{s}} \Xi_{2} \mathbb{J}_{1}\left(a_{\mathrm{s}} \Xi_{2}\right)-\hat{A}_{2} \hat{\mathscr{C}}_{\mathrm{e}} a_{\mathrm{s}} \Xi_{2, \mathrm{e}} \mathbb{J}_{1}\left(a_{\mathrm{s}} \Xi_{2, \mathrm{e}}\right)\right] \\
\mathscr{I}_{\mathrm{w}, 2}=-\log _{2}(e) \sum_{\boldsymbol{\eta} \in \Phi} \kappa_{\boldsymbol{\eta}}\left[\check{A}_{2} \Xi_{2} \mathbb{J}_{2}\left(\Xi_{2}\right)-\check{A}_{2} \check{\mathscr{C}}_{\mathrm{e}} \Xi_{2, \mathrm{e}} \mathbb{J}_{2}\left(\Xi_{2, \mathrm{e}}\right)+\hat{A}_{2} a_{\mathrm{s}} \Xi_{2} \mathbb{J}_{2}\left(a_{\mathrm{s}} \Xi_{2}\right)-\hat{A}_{2} \hat{\mathscr{C}}_{\mathrm{e}} a_{\mathrm{s}} \Xi_{2, \mathrm{e}} \mathbb{J}_{2}\left(a_{\mathrm{s}} \Xi_{2, \mathrm{e}}\right)\right] \\
\mathscr{I}_{\mathrm{w}, 3}=-\log _{2}(e) \sum_{\boldsymbol{\eta} \in \Phi} \kappa_{\boldsymbol{\eta}}\left\{1-\exp \left(-B_{\boldsymbol{\eta}} \Theta\right)\right\}\left[\check{\mathcal{A}}_{2} \check{C}_{\mathrm{e}} \Xi_{2, \mathrm{e}} \mathbb{J}_{1}\left(\Xi_{2, \mathrm{e}}\right)+\hat{\mathcal{A}}_{2} \hat{C}_{\mathrm{e}} a_{\mathrm{s}} \Xi_{2, \mathrm{e}} \mathbb{J}_{1}\left(a_{\mathrm{s}} \Xi_{2, \mathrm{e}}\right)\right] \\
\mathscr{I}_{\mathrm{w}, 4}=-\log _{2}(e) \sum_{\boldsymbol{\eta} \in \Phi} \kappa_{\boldsymbol{\eta}}\left[\check{\mathcal{A}}_{2} \check{C}_{\mathrm{e}} \Xi_{2, \mathrm{e}} \mathbb{J}_{2}\left(\Xi_{2, \mathrm{e}}\right)+a_{\mathrm{s}} \hat{\mathcal{A}}_{2} \hat{C}_{\mathrm{e}} \Xi_{2, \mathrm{e}} \mathbb{J}_{2}\left(a_{\mathrm{s}} \Xi_{2, \mathrm{e}}\right)\right]
\end{gathered}
$$

between the numerical and analytical results (for the case of NOMA) verifies the correctness of the derived closed-form expressions.

Fig. 3 shows a comparison of the ESSR for the NOMA and OMA systems. Note that the NOMA system outperforms its OMA-based counterpart by achieving a significantly higher ESSR. The ESSR for the NOMA/OMA system first increases with an increase of the value of $I_{\mathrm{p}}$ (which we refer to the interference-constrained regime) and then saturates for larger value of $I_{\mathrm{p}}$ (which we refer to as the power-constrained regime). Interestingly, in the interference-constrained regime, the ESSR of the NOMA system remains the same irrespective of the value of $P_{\max }$, whereas, in the power-constrained regime, the ESSR is independent of the value of $I_{\mathrm{p}}$. A similar behavior is evident for the OMA system. It is noteworthy that for the system parameters considered in Fig. 3, $\min \left(P_{\max }, \frac{I_{\mathrm{p}}}{\mathbb{E}\left\{\tilde{g}_{\mathrm{p}}\right\}}\right)=\min \left(P_{\max }, \frac{I_{\mathrm{p}}}{\tilde{\Omega}_{\mathrm{p}}}\right)$ becomes constant $\left(=P_{\max }\right)$ w.r.t $I_{p}$ at $I_{\mathrm{p}} \gtrsim 0 \mathrm{~dB}$ for $P_{\max }=40 \mathrm{~dB}$, at $I_{\mathrm{p}} \gtrsim 10 \mathrm{~dB}$ for $P_{\max }=50 \mathrm{~dB}$, and at $I_{\mathrm{p}} \gtrsim 20 \mathrm{~dB}$ for $P_{\max }=60 \mathrm{~dB}$. Therefore, the ESSR for both the NOMA and 


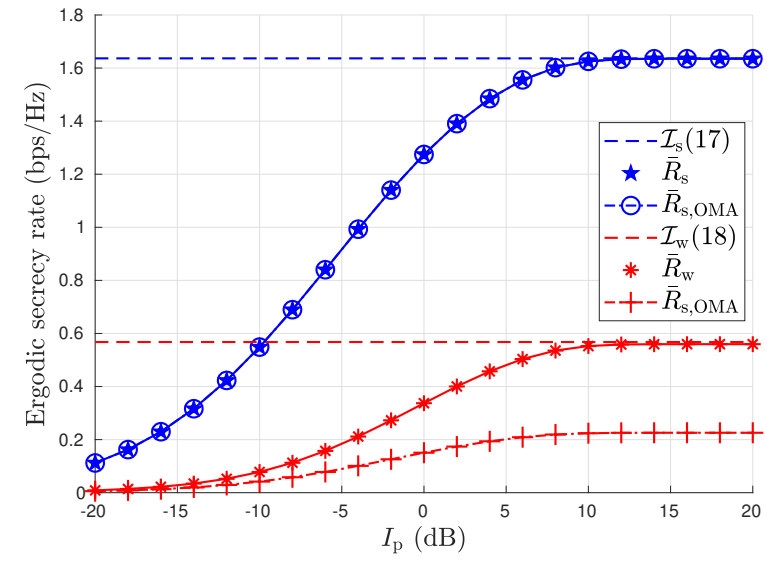

Fig. 2. Comparison of ESR for NOMA and OMA systems for $P_{\max }=$ $50 \mathrm{~dB}$.

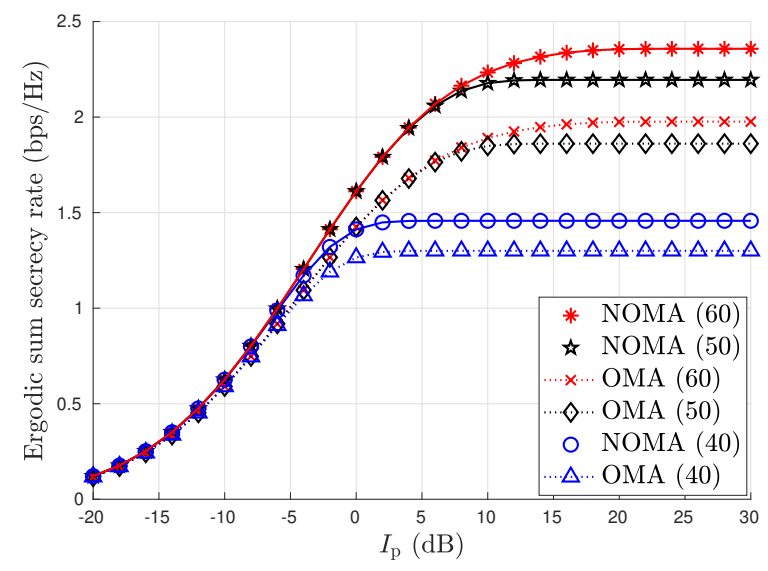

Fig. 3. Comparison of ESSR for NOMA and OMA systems. The number in the parentheses denotes $P_{\max }$ in $\mathrm{dB}$.

OMA systems becomes constant w.r.t. $I_{\mathrm{p}}$ (power-constrained regime) at $I_{\mathrm{p}} \gtrsim\{0,10,20\} \mathrm{dB}$ for $P_{\max }=\{40,50,60\} \mathrm{dB}$.

In Fig. 4, we investigate the effect of channel estimation error on the ESSR of the NOMA and OMA systems. It can be noted from the figure that the ESSR for both the NOMA and OMA systems decreases with an increase in the value of $\sigma_{\epsilon}^{2}$. This implies that a decrease in the accuracy of channel estimation has an adverse effect on the system performance. The effect of the interference-constrained regime is also clearly evident from the figure, as the value of ESSR at $I_{\mathrm{p}}=0 \mathrm{~dB}$ is the same for $P_{\max }=50 \mathrm{~dB}$ and $P_{\max }=60 \mathrm{~dB}$. However, for a large value of $I_{\mathrm{p}}(10 \mathrm{~dB})$, the benefit of a larger $P_{\max }$ can be noted from the figure.

Fig. 5 shows the effect of the number and distance of PRs on the ESSR of the NOMA system. In this figure, three different cases are studied. In Case I, we consider 2 PRs both located at a distance of $200 \mathrm{~m}$ from the ST; in Case II, we consider 4 PRs located at $\{200,205,210,215\} \mathrm{m}$ from the ST, and in Case III, we consider 10 PRs, all located at a distance of $200 \mathrm{~m}$. Moreover, for all of the cases, we consider two different values of transmit power: $P_{\max }=40 \mathrm{~dB}$ and $60 \mathrm{~dB}$.

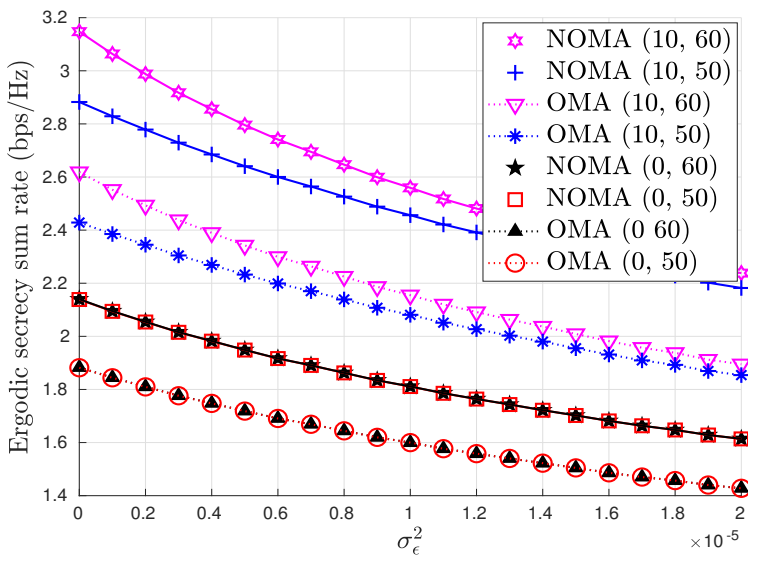

Fig. 4. Effect of channel estimation error on the ESSR. The numbers in the parentheses denote $\left(I_{\mathrm{p}}, P_{\max }\right)$ in $\mathrm{dB}$.

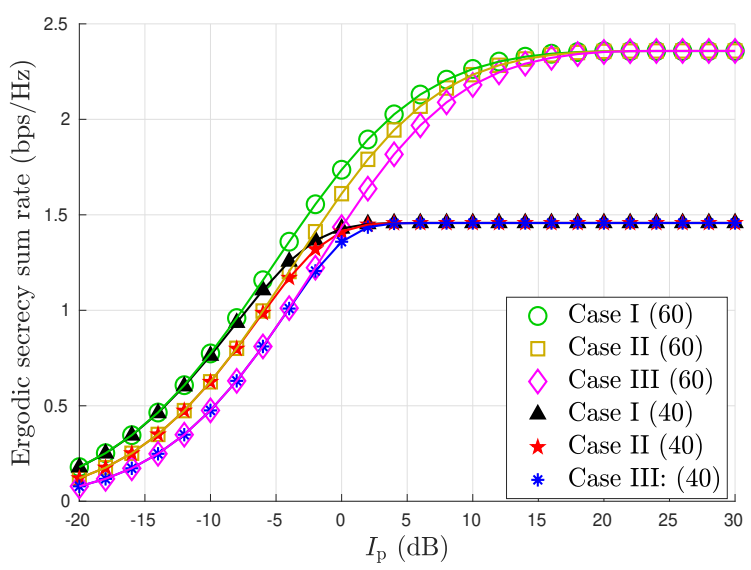

Fig. 5. Effect of the number of PRs on the ESSR of the NOMA system. The number in the parentheses denotes $P_{\max }$ in $\mathrm{dB}$.

It is evident from the figure that in the interference-constrained regime (i.e., for small values of $I_{\mathrm{p}}$ ), an increase in the number of PRs results in a reduction of the ESSR. However, in this regime, no benefit is observed from a higher $P_{\max }$ budget. On the other hand, in the power-constrained regime (i.e., for large values of $I_{p}$ ), the ESSR for all the cases becomes the same, irrespective of the number/location of PRs. This occurs due to the reason that in the power-constrained regime, the secrecy performance becomes independent of the ST-PR link quality as well as the interference constraint at the PRs. However, a larger $P_{\max }$ turns out to be clearly beneficial in this regime.

\section{CONCLUSION}

In this paper, we have presented the ergodic secrecy rate analysis of a two-user downlink NOMA system in an underlay spectrum sharing scenario consisting of multiple primary-user receivers in the presence of channel estimation error. Exact and asymptotic closed-form expressions for the ESSR of the NOMA system were derived. Our results confirmed that the NOMA system outperforms its OMA-based counterpart in terms of the ESSR. More interestingly, the results confirmed 
that no benefit is obtained in terms of the ESSR from a higher power budget at the ST in the interference-constrained regime, whereas, in the power-constrained regime, the ESSR becomes independent of the number of PRs as well as the quality of the ST-PR links. The results also showed that a larger channel estimation error results in a reduced secrecy rate. Finally, the asymptotic analysis demonstrated that the ESSR of the NOMA system becomes independent of the value of peak tolerable interference and therefore, the slope of the ESSR w.r.t. the peak tolerable interference becomes zero.

\section{APPENDIX A}

\section{PROOF OF THEOREM 1}

Using (1), we have

$$
\begin{aligned}
& \bar{R}_{\mathrm{s}}=\mathbb{E}\left\{\max \left\{\log _{2}\left(1+\hat{g}_{\mathrm{s}} P\right)-\log _{2}\left(1+\hat{g}_{\mathrm{e}} P\right), 0\right\}\right\} \\
= & \int_{y=0}^{\Theta} \int_{x=\sigma_{\epsilon}^{2}}^{\infty} \log _{2}\left(1+P_{\max } x\right) f_{\hat{g}_{\mathrm{s}}}(x) \mathscr{F}_{\hat{g}_{\mathrm{e}}}\left(\sigma_{\epsilon}^{2}, x\right) f_{\tilde{g}_{\mathrm{p}}}(y) \mathrm{d} x \mathrm{~d} y \\
+ & \int_{y=\Theta}^{\infty} \int_{x=\sigma_{\epsilon}^{2}}^{\infty} \log _{2}\left(1+I_{\mathrm{p}} \frac{x}{y}\right) f_{\hat{g}_{\mathrm{s}}}(x) \mathscr{F}_{\hat{g}_{\mathrm{e}}}\left(\sigma_{\epsilon}^{2}, x\right) f_{\tilde{g}_{\mathrm{p}}}(y) \mathrm{d} x \mathrm{~d} y \\
- & \int_{y=0}^{\Theta} \int_{x=\sigma_{\epsilon}^{2}}^{\infty} \log _{2}\left(1+P_{\max } x\right) f_{\hat{g}_{\mathrm{e}}}(x) \mathcal{F}_{\hat{g}_{\mathrm{s}}}(x) f_{\tilde{g}_{\mathrm{p}}}(y) \mathrm{d} x \mathrm{~d} y \\
- & \int_{y=\Theta}^{\infty} \int_{x=\sigma_{\epsilon}^{2}}^{\infty} \log _{2}\left(1+I_{\mathrm{p}} \frac{x}{y}\right) f_{\hat{g}_{\mathrm{e}}}(x) \mathcal{F}_{\hat{g}_{\mathrm{s}}}(x) f_{\tilde{g}_{\mathrm{p}}}(y) \mathrm{d} x \mathrm{~d} y \\
\triangleq & \mathscr{I}_{\mathrm{s}, 1}+\mathscr{I}_{s, 2}-\mathscr{I}_{s, 3}-\mathscr{I}_{s, 4} .
\end{aligned}
$$

Solving for $\mathscr{I}_{\mathrm{s}, 1}$, using the expressions for $f_{\hat{g}_{\mathrm{s}}}(x), \mathscr{F}_{\hat{g}_{\mathrm{e}}}\left(\sigma_{\epsilon}^{2}, x\right)$ and $f_{\tilde{g}_{\mathrm{p}}}(y)$ yields

$$
\begin{gathered}
\mathscr{I}_{\mathrm{s}, 1}=-\log _{2}(e) \sum_{\ell \in\{1,2,3\}} \sum_{\boldsymbol{\eta} \in \Phi} \hat{A}_{\ell} B_{\boldsymbol{\eta}} \kappa_{\boldsymbol{\eta}} \\
\times\left[\int_{y=0}^{\Theta} \int_{x=\sigma_{\epsilon}^{2}}^{\infty} \ln \left(1+P_{\max } x\right) \exp \left(\frac{-x}{a_{\mathrm{s}} \Xi_{\ell}}\right) \exp \left(-B_{\boldsymbol{\eta}} y\right) \mathrm{d} x \mathrm{~d} y\right. \\
\left.-\hat{\mathscr{C}}_{\mathrm{e}} \int_{y=0}^{\Theta} \int_{x=\sigma_{\epsilon}^{2}}^{\infty} \ln \left(1+P_{\max } x\right) \exp \left(\frac{-x}{a_{\mathrm{s}} \Xi_{\ell, \mathrm{e}}}\right) \exp \left(-B_{\boldsymbol{\eta}} y\right) \mathrm{d} x \mathrm{~d} y\right] \\
=-\log _{2}(e) \sum_{\ell \in\{1,2,3\}} \sum_{\boldsymbol{\eta} \in \Phi} \hat{A}_{\ell} \kappa_{\boldsymbol{\eta}}\left\{1-\exp \left(-B_{\boldsymbol{\eta}} \Theta\right)\right\} \\
\times\left[\int_{x=\sigma_{\epsilon}^{2}}^{\infty} \ln \left(1+P_{\max } x\right) \exp \left(\frac{-x}{a_{\mathrm{s}} \Xi_{\ell}}\right) \mathrm{d} x\right. \\
\left.\quad-\hat{\mathscr{C}}_{\mathrm{e}} \int_{x=\sigma_{\epsilon}^{2}}^{\infty} \ln \left(1+P_{\max } x\right) \exp \left(\frac{-x}{a_{\mathrm{s}} \Xi_{\ell, \mathrm{e}}}\right) \mathrm{d} x\right] .
\end{gathered}
$$

Computing the integrals above using integration by parts and with some algebraic manipulations, we arrive at the closedform expression for $\mathscr{I}_{\mathrm{s}, 1}$ is given by (3). Similarly, for $\mathscr{I}_{\mathrm{s}, 3}$, we have

$$
\begin{aligned}
& \mathscr{I}_{\mathrm{s}, 3}=-\log _{2}(e) \hat{C}_{\mathrm{e}} \sum_{\ell \in\{1,2,3\}} \sum_{\boldsymbol{\eta} \in \Phi} \hat{\mathcal{A}}_{\ell} B_{\boldsymbol{\eta}} \kappa_{\boldsymbol{\eta}} \\
& \times \int_{y=0}^{\Theta} \int_{x=\sigma_{\epsilon}^{2}}^{\infty} \ln \left(1+P_{\max } x\right) \exp \left(\frac{-x}{a_{\mathrm{s}} \Xi_{\ell, \mathrm{e}}}\right) \exp \left(-B_{\boldsymbol{\eta}} y\right) \mathrm{d} x \mathrm{~d} y
\end{aligned}
$$

$$
\begin{gathered}
=-\log _{2}(e) \hat{C}_{\mathrm{e}} \sum_{\ell \in\{1,2,3\}} \sum_{\boldsymbol{\eta} \in \Phi} \hat{\mathcal{A}}_{\ell} \kappa_{\boldsymbol{\eta}}\left\{1-\exp \left(-B_{\boldsymbol{\eta}} \Theta\right)\right\} \\
\times \int_{x=\sigma_{\epsilon}^{2}}^{\infty} \ln \left(1+P_{\max } x\right) \exp \left(\frac{-x}{a_{\mathrm{s}} \Xi_{\ell, \mathrm{e}}}\right) \mathrm{dx} .
\end{gathered}
$$

A closed-form expression for $\mathscr{I}_{\mathrm{s}, 3}$ can be obtained by following the steps similar to those used to obtain $\mathscr{I}_{\mathrm{s}, 1}$, and is given in (5). Now solving for $\mathscr{I}_{\mathrm{s}, 4}$, we have

$$
\begin{aligned}
& \mathscr{I}_{s, 4}=-\log _{2}(e) \hat{C}_{\mathrm{e}} \sum_{\ell \in\{1,2,3\}} \sum_{\boldsymbol{\eta} \in \Phi} \hat{\mathcal{A}}_{\ell} B_{\boldsymbol{\eta}} \kappa_{\boldsymbol{\eta}} \int_{y=\Theta}^{\infty} \\
& {\left[\int_{x=\sigma_{\epsilon}^{2}}^{\infty} \ln \left(1+\frac{I_{\mathrm{p}}}{y} x\right) \exp \left(\frac{-x}{a_{\mathrm{s}} \Xi_{\ell, \mathrm{e}}}\right) \mathrm{d} x\right] \exp \left(-B_{\boldsymbol{\eta}} y\right) \mathrm{d} y .}
\end{aligned}
$$

Integrating the preceding expression w.r.t. $x$ using integration by parts and using a few algebraic manipulations yields

$$
\begin{aligned}
& \mathscr{I}_{\mathrm{s}, 4}=-\log _{2}(e) \hat{C}_{\mathrm{e}} \sum_{\ell \in\{1,2,3\}} \sum_{\boldsymbol{\eta} \in \Phi} \hat{\mathcal{A}}_{\ell} B_{\boldsymbol{\eta}} \kappa_{\boldsymbol{\eta}} a_{\mathrm{s}} \Xi_{\ell, \mathrm{e}} \int_{y=\Theta}^{\infty} \\
& \times\left[\exp \left(\frac{-\sigma_{\epsilon}^{2}}{a_{\mathrm{s}} \Xi_{\ell, \mathrm{e}}}\right) \ln \left(1+\frac{I_{\mathrm{p}} \sigma_{\epsilon}^{2}}{y}\right)+\exp \left(\frac{y}{a_{\mathrm{s}} I_{\mathrm{p}} \Xi_{\ell, \mathrm{e}}}\right)\right. \\
& \left.\times \mathrm{E}_{1}\left(\frac{1}{a_{\mathrm{s}} \Xi_{\ell, \mathrm{e}}}\left(\frac{y}{I_{\mathrm{p}}}+\sigma_{\epsilon}^{2}\right)\right)\right] \exp \left(-B_{\boldsymbol{\eta}} y\right) \mathrm{d} y .
\end{aligned}
$$

Now solving the integral w.r.t $y$ using integration by parts, a closed-form expression for $\mathscr{I}_{\mathrm{s}, 4}$ is given in (6). Next, for $\mathscr{I}_{\mathrm{s}, 2}$, we have

$$
\begin{aligned}
& \mathscr{I}_{\mathrm{s}, 2}=-\log _{2}(e) \sum_{\ell \in\{1,2,3\}} \sum_{\boldsymbol{\eta} \in \Phi} \hat{A}_{\ell} B_{\boldsymbol{\eta}} \kappa_{\boldsymbol{\eta}} \int_{y=\Theta}^{\infty} \int_{x=\sigma_{\epsilon}^{2}}^{\infty} \ln \left(1+\frac{I_{\mathrm{p}}}{y} x\right) \\
& \times \exp \left(\frac{-x}{a_{\mathrm{s}} \Xi_{\ell}}\right)\left\{1-\hat{\mathscr{C}}_{\mathrm{e}} \exp \left(\frac{-x}{a_{\mathrm{s}} \tilde{\Omega}_{\mathrm{e}}}\right)\right\} \exp \left(-B_{\boldsymbol{\eta}} y\right) \mathrm{d} x \mathrm{~d} y .
\end{aligned}
$$

Solving the integrals above by following the steps similar to those used for $\mathscr{I}_{\mathrm{s}, 4}$, a closed-form expression for $\mathscr{I}_{\mathrm{s}, 2}$ is given by (4). This concludes the proof.

\section{APPENDIX B} PROOF OF THEOREM 2

Using (9), we have

$$
\begin{aligned}
\bar{R}_{\mathrm{w}}=\mathbb{E}\left\{\operatorname { m a x } \left\{\log _{2}\left(\frac{1+\left(\tilde{g}_{\mathrm{w}}+\sigma_{\epsilon}^{2}\right) P}{1+\left(a_{\mathrm{s}} \tilde{g}_{\mathrm{w}}+\sigma_{\epsilon}^{2}\right) P}\right)\right.\right. & \left.\left.-\log _{2}\left(\frac{1+\left(\tilde{g}_{\mathrm{e}}+\sigma_{\epsilon}^{2}\right) P}{1+\left(a_{\mathrm{s}} \tilde{g}_{\mathrm{e}}+\sigma_{\epsilon}^{2}\right) P}\right), 0\right\}\right\} \\
= & {\left[\int_{y=0}^{\Theta} \int_{x=\sigma_{\epsilon}^{2}}^{\infty} \log _{2}\left(1+x P_{\max }\right) f_{\check{g}_{\mathrm{w}}}(x) \mathscr{F}_{\check{g}_{\mathrm{e}}}(x) f_{\tilde{g}_{\mathrm{p}}}(y) \mathrm{d} x \mathrm{~d} y\right.} \\
- & \left.\int_{y=0}^{\Theta} \int_{x=\sigma_{\epsilon}^{2}}^{\infty} \log _{2}\left(1+x P_{\max }\right) f_{\hat{g}_{\mathrm{w}}}(x) \mathscr{F}_{\hat{g}_{\mathrm{e}}}(x) f_{\tilde{g}_{\mathrm{p}}}(y) \mathrm{d} x \mathrm{~d} y\right] \\
+ & {\left[\int_{y=\Theta}^{\infty} \int_{x=\sigma_{\epsilon}^{2}}^{\infty} \log _{2}\left(1+I_{\mathrm{p}} \frac{x}{y}\right) f_{\check{g}_{\mathrm{w}}}(x) \mathscr{F}_{\check{g}_{\mathrm{e}}}(x) f_{\tilde{g}_{\mathrm{p}}}(y) \mathrm{d} x \mathrm{~d} y\right.} \\
- & \left.\int_{y=\Theta}^{\infty} \int_{x=\sigma_{\epsilon}^{2}}^{\infty} \log _{2}\left(1+I_{\mathrm{p}} \frac{x}{y}\right) f_{\hat{g}_{\mathrm{w}}}(x) \mathscr{F}_{\hat{g}_{\mathrm{e}}}(x) f_{\tilde{g}_{\mathrm{p}}}(y) \mathrm{d} x \mathrm{~d} y\right]
\end{aligned}
$$




$$
\begin{aligned}
& -\left[\int_{y=0}^{\Theta} \int_{x=\sigma_{\epsilon}^{2}}^{\infty} \log _{2}\left(1+x P_{\max }\right) f_{\check{g}_{\mathrm{e}}}(x) \mathcal{F}_{\breve{g}_{\mathrm{w}}}(x) f_{\tilde{g}_{\mathrm{p}}}(y) \mathrm{d} x \mathrm{~d} y\right. \\
& \left.-\int_{y=0}^{\Theta} \int_{x=\sigma_{\epsilon}^{2}}^{\infty} \log _{2}\left(1+x P_{\max }\right) f_{\hat{g}_{\mathrm{e}}}(x) \mathcal{F}_{\hat{g}_{\mathrm{w}}}(x) f_{\tilde{g}_{\mathrm{p}}}(y) \mathrm{d} x \mathrm{~d} y\right] \\
& -\left[\int_{y=\Theta}^{\infty} \int_{x=\sigma_{\epsilon}^{2}}^{\infty} \log _{2}\left(1+I_{\mathrm{p}} \frac{x}{y}\right) f_{\check{g}_{\mathrm{e}}}(x) \mathcal{F}_{\check{g}_{\mathrm{w}}}(x) f_{\tilde{g}_{\mathrm{p}}}(y) \mathrm{d} x \mathrm{~d} y\right. \\
& \left.-\int_{y=\Theta}^{\infty} \int_{x=\sigma_{\epsilon}^{2}}^{\infty} \log _{2}\left(1+I_{\mathrm{p}} \frac{x}{y}\right) f_{\hat{g}_{\mathrm{e}}}(x) \mathcal{F}_{\hat{g}_{\mathrm{w}}}(x) f_{\tilde{g}_{\mathrm{p}}}(y) \mathrm{d} x \mathrm{~d} y\right] \\
& \triangleq \mathscr{I}_{\mathrm{w}, 1}+\mathscr{I}_{\mathrm{w}, 2}-\mathscr{I}_{\mathrm{w}, 3}-\mathscr{I}_{\mathrm{w}, 4} .
\end{aligned}
$$

Following a similar line of argument as given in Appendix A, closed-form expressions for $\mathscr{I}_{\mathrm{w}, 1}, \mathscr{I}_{\mathrm{w}, 2}, \mathscr{I}_{\mathrm{w}, 3}$ and $\mathscr{I}_{\mathrm{w}, 4}$ are given by (11) - (14). This completes the proof.

\section{REFERENCES}

[1] M. Vaezi, Z. Ding, and H. Poor, Multiple Access Techniques for $5 G$ Wireless Networks and Beyond. Springer International Publishsing, 2018.

[2] A. Goldsmith, S. A. Jafar, I. Maric, and S. Srinivasa, "Breaking spectrum gridlock with cognitive radios: An information theoretic perspective," Proc. of the IEEE, vol. 97, no. 5, pp. 894-914, May 2009.

[3] L. Lv, J. Chen, Q. Ni, Z. Ding, and H. Jiang, "Cognitive non-orthogonal multiple access with cooperative relaying: A new wireless frontier for 5G spectrum sharing," IEEE Commun. Mag., vol. 56, no. 4, pp. 188-195, Apr 2018.

[4] V. Kumar, B. Cardiff, and M. F. Flanagan, "Fundamental limits of spectrum sharing for NOMA-based cooperative relaying under a peak interference constraint," IEEE Trans. Commun., vol. 67, no. 12, pp. 8233-8246, 2019.
[5] V. Kumar, Z. Ding, and M. Flanagan, "On the performance of downlink NOMA in underlay spectrum sharing," IEEE Trans. Veh. Technol., to appear.

[6] L. Zhao, X. Zhang, J. Chen, and L. Zhou, "Physical layer security in the age of artificial intelligence and edge computing," IEEE Wireless Commun., vol. 27, no. 5, pp. 174-180, 2020.

[7] H. Lei, Z. Yang, K. Park, I. S. Ansari, Y. Guo, G. Pan, and M. Alouini, "Secrecy outage analysis for cooperative NOMA systems with relay selection schemes," IEEE Trans. Commun., vol. 67, no. 9, pp. 62826298, 2019.

[8] L. Lv, H. Jiang, Z. Ding, L. Yang, and J. Chen, "Secrecy-enhancing design for cooperative downlink and uplink NOMA with an untrusted relay," IEEE Trans. Commun., vol. 68, no. 3, pp. 1698-1715, 2020.

[9] C. Guo, L. Zhao, C. Feng, Z. Ding, and H. M. Wang, "Secrecy performance of NOMA systems with energy harvesting and full-duplex relaying," IEEE Trans. Veh. Technol., vol. 69, no. 10, pp. 12 301-12 305, 2020.

[10] X. Liu, K. Zheng, L. Fu, X. Liu, X. Wang, and G. Dai, "Energy efficiency of secure cognitive radio networks with cooperative spectrum sharing," IEEE Trans. Mob. Comp., vol. 18, no. 2, pp. 305-318, 2019.

[11] J. Zhang, C. Kundu, O. A. Dobre, E. Garcia-Palacios, and N. Vo, "Secrecy performance of small-cell networks with transmitter selection and unreliable backhaul under spectrum sharing environment," IEEE Trans. Veh. Technol., vol. 68, no. 11, pp. 10895-10908, 2019.

[12] S. Timilsina, G. A. Aruma Baduge, and R. F. Schaefer, "Secure communication in spectrum-sharing massive MIMO systems with active eavesdropping," IEEE Trans. Cognitive Commun. Net., vol. 4, no. 2, pp. 390-405, 2018

[13] T. Q. Duong, X. Zhou, and H. V. Poor, Trusted communications with physical layer security for $5 G$ and beyond. Institution of Engineering \& Technology, 2017.

[14] A. Hyadi, M. Benjillali, M. Alouini, and D. B. da Costa, "Performance analysis of underlay cognitive multihop regenerative relaying systems with multiple primary receivers," IEEE Trans. Wireless Commun. vol. 12, no. 12, pp. 6418-6429, 2013. 